\title{
Maximum Likelihood for Dual Varieties
}

\author{
Jose Israel Rodriguez*
}

19 May 2014

\begin{abstract}
Maximum likelihood estimation (MLE) is a fundamental computational problem in statistics. In this paper, MLE for statistical models with discrete data is studied from an algebraic statistics viewpoint. A reformulation of the MLE problem in terms of dual varieties and conormal varieties will be given. With this description, the dual likelihood equations and the dual MLE problem are defined. We show that solving the dual MLE problem yields solutions to the MLE problem, so we can solve the MLE problem without ever determining the defining equations of the model.
\end{abstract}

\section{Introduction}

Maximum likelihood estimation (MLE) is a fundamental problem in statistics that has been extensively studied from an algebraic viewpoint [3, 4, 5, 9, 10, 12]. We continue to follow an algebraic approach to MLE in this paper considering statistical models for discrete data in the probability simplex as irreducible varieties $X$ in complex projective space $\mathbb{P}^{n}$.

An algebraic statistical model $X$ in $\mathbb{P}^{n}$ will be defined by the vanishing of homogeneous polynomials in the unknowns $p_{0}, p_{1}, \ldots, p_{n}$. We assume that $X$ is an irreducible generically reduced variety. When the coordinates $p_{0}, p_{1}, \ldots, p_{n}$ of a point $p$ in $X$ are positive and sum to one, we interpret $p$ as a probability distribution, where the probability of observing event $i$ is $p_{i}$. We let $u=\left(u_{0}, u_{1}, \ldots, u_{n}\right) \in\left(\mathbb{C}^{*}\right)^{n+1}$ be a vector of length $n+1$ called data. When each entry $u_{i}$ of the vector is a positive integer we interpret $u_{i}$ as the number of observations of event $i$. We use the notation

$$
u_{+}:=u_{0}+\cdots+u_{n} \text { and } p_{+}:=p_{0}+\cdots+p_{n},
$$

always assuming $u_{+} \neq 0$.

\footnotetext{
*Department of Mathematics, University of California at Berkeley, Berkeley, CA 94720; jo.ro@berkeley.edu.
} 
The likelihood function for $u$ is defined as

$$
l_{u}(p):=p_{0}^{u_{0}} p_{1}^{u_{1}} \cdots p_{n}^{u_{n}} / p_{+}^{u_{+}} .
$$

When $u$ and $p$ are interpreted as data and a probability distribution respectively, the likelihood of observing $u$ with respect to the distribution $p$ is $l_{u}(p)$ divided by a multinomial coefficient depending only on $u$.

For fixed data $u$, to determine local maxima of $l_{u}(p)$ on a statistical model and give a solution to the MLE problem, we determine all complex critical points of $l_{u}(p)$ restricted to $X$. Of these critical points, we find the one with positive coordinates and greatest likelihood to determine the maximum likelihood estimator $\hat{p}$. The (algebraic) maximum likelihood estimation problem is solved by determining all critical points of $l_{u}(p)$ on $X$ and maximizing $l_{u}(p)$ on this set.

To find the complex critical points, we determine when the gradient of $l_{u}(p)$ is orthogonal to the tangent space of $X$ at $p$. So the set of critical points is

$$
\left\{p \in X_{\text {reg }} \text { such that } \nabla l_{u}(p) \perp T_{p} X\right\} \text {. }
$$

The gradient of the likelihood function equals $\left[\frac{u_{0}}{p_{0}}-\frac{u_{+}}{p_{+}}, \frac{u_{1}}{p_{1}}-\frac{u_{+}}{p_{+}}, \ldots, \frac{u_{n}}{p_{n}}-\frac{u_{+}}{p_{+}}\right]$, up to scaling by $l_{u}(p) / p_{+}^{u+}$. So the critical points of $l_{u}(p)$ are $p \in X_{\text {reg }}$ such that

$$
\left[\frac{u_{0}}{p_{0}}-\frac{u_{+}}{p_{+}}, \frac{u_{1}}{p_{1}}-\frac{u_{+}}{p_{+}}, \ldots, \frac{u_{n}}{p_{n}}-\frac{u_{+}}{p_{+}}\right] \perp T_{p}(X),
$$

implicitly forcing the condition $p_{0} p_{1} \cdots p_{n}\left(p_{0}+\cdots+p_{n}\right) \neq 0$.

Definition 1. Given an algebraic statistical model $X$ in $\mathbb{P}^{n}$, the maximum likelihood degree (ML degree) of $X$ is the number of critical points of $l_{u}(p)$ restricted to $X$ for generic choices of data $u$,

$$
\operatorname{MLdegree}(X)=\#\left\{p \in X: \nabla l_{u}(p) \perp T_{p}(X)\right\} .
$$

The main result of this paper is to give a formulation that relates maximum likelihood estimation to a conormal variety derived from $X$ [Theorem 4 . With this perspective, we use the dual likelihood equations [Theorem 8 , to solve the MLE problem for $X$ when only given the defining equations of its dual variety $X^{*}$.

The computations in this paper were done using Bertini [1] and Macaulay2 [7].

\section{Maximum Likelihood Estimation}

In this section, we consider an algebraic statistical model $X$ in $\mathbb{P}^{n}$ and will define $X^{\prime}$ to be an embedding of $X$ in $\mathbb{P}^{n+1}$. We will present our first result in Theorem 4 giving a 
formulation of the MLE problem in terms of conormal varieties and dual varieties. In Corollary 1 we give a bijection between critical points of the likelihood function on two different varieties. In Corollary 2 we give equations to solve the MLE problem if we have equations that define a conormal variety. We will also recall how to compute conormal varieties and dual varieties of $X$ and $X^{\prime}$.

Let $X \subset \mathbb{P}^{n}$ be a codimension $c$ algebraic statistical model defined by homogenous polynomials $f_{1}, f_{2}, \ldots, f_{k}$. We let $\operatorname{Jac}(X)$ denote the $k \times(n+1)$ matrix of partial derivatives of $f_{1}, \ldots, f_{k}$ with respect to $p_{0}, \ldots, p_{n}$, and we say this is the Jacobian of $X$.

To keep track of the sum of the coordinates $p_{0}, p_{1}, \ldots, p_{n}$ we introduce the coordinate $p_{s}$ and a hyperplane in $\mathbb{P}^{n+1}$ defined by the vanishing of the polynomial

$$
H(p):=-p_{0}-p_{1}+\cdots-p_{n}+p_{s} .
$$

If $X$ is defined by $f_{1}, \ldots, f_{k}$ then $X^{\prime}$ in the coordinates $p_{0}, p_{1}, \ldots, p_{n}, p_{s}$ is defined by the vanishing of $f_{1}, \ldots, f_{k}$ and $H$. With this definition, we get the following proposition.

Proposition 1. If $X$ is defined by the homogeneous polynomials $f_{1}, f_{2}, \ldots, f_{k}$ then the Jacobian of $X^{\prime}$ is given by the $(k+1) \times(n+2)$-matrix

$$
\operatorname{Jac}\left(X^{\prime}\right)=\left[\begin{array}{ccccc}
-1 & -1 & \cdots & -1 & 1 \\
& & & & 0 \\
& \operatorname{Jac}(X) & & \vdots \\
& & & & 0
\end{array}\right] .
$$

The important fact about the construction of $X^{\prime}$ is that there is a bijection between the critical points of the function $l_{u}(p)$ on $X$ and the critical points of the monomial

$$
l_{u}^{\prime}(p):=p_{0}^{u_{0}} p_{1}^{u_{1}} \cdots p_{n}^{u_{n}} p_{s}^{-u_{+}} \text {on } X^{\prime}
$$

given by Lemma 2

By a slight abuse of notation the " $p$ " in $l_{u}(p)$ and the " $p$ " in $l_{u}^{\prime}(p)$ represent two different things. The first $p$ represents a point $\left[p_{0}: p_{1}: \cdots: p_{n}\right] \in X$, while the second represents a point $\left[p_{0}: p_{1}: \cdots: p_{n}: p_{s}\right] \in X^{\prime}$.

Lemma 2. There is a bijection between the critical points of the function $l_{u}(p)$ on $X$ and the critical points of $l_{u}^{\prime}(p)$ on $X^{\prime}$. Under this bijection, $\left[p_{0}: p_{1}: \cdots: p_{n}\right] \in \mathbb{P}^{n}$ is a critical point of $l_{u}(p)$ on $X$ if and only if $\left[p_{0}: p_{1}: \cdots: p_{n}: p_{s}\right] \in \mathbb{P}^{n+1}$ is a critical point of $l_{u}^{\prime}(p)$ on $X^{\prime}$.

Proof. To prove this we need to show that

$$
\left[p_{0}: \cdots: p_{n}: p_{s}\right] \in X_{r e g}^{\prime} \text { satisfies } \nabla l_{u}^{\prime}(p) \perp T_{p} X^{\prime}
$$

if and only if

$$
\left[p_{0}: \cdots: p_{n}\right] \in X_{\text {reg }} \text { satisfies } \nabla l_{u}(p) \perp T_{p} X \text {. }
$$


By Proposition 1, it follows $\left[p_{0}: \cdots: p_{n}: p_{s}\right] \in X_{r e g}^{\prime}$ if and only if $\left[p_{0}: \cdots: p_{n}\right] \in X_{r e g}$, so it remains to show that $\nabla l_{u}^{\prime}(p) \perp T_{p} X^{\prime}$ if and only if $\nabla l_{u}(p) \perp T_{p} X$. So we need to show that $\nabla l_{u}^{\prime}(p)$ being in the row space of $\operatorname{Jac}\left(X^{\prime}\right)$ implies that $\nabla l_{u}(p)$ is in the row space of $\operatorname{Jac}(X)$ and vice versa. To see this, observe that

$$
\begin{aligned}
& {\left[\begin{array}{c}
\nabla l_{u}^{\prime}(p) \\
\operatorname{Jac}\left(X^{\prime}\right)
\end{array}\right]\left[\begin{array}{cccc}
1 & & & \\
& \ddots & & \\
& & 1 & \\
1 & \cdots & 1 & 1
\end{array}\right]=} \\
& {\left[\begin{array}{ccccc}
\frac{u_{0}}{p_{0}}-\frac{u_{+}}{p_{s}} & \frac{u_{1}}{p_{1}}-\frac{u_{+}}{p_{s}} & \cdots & \frac{u_{n}}{p_{n}}-\frac{u_{+}}{p_{s}} & -\frac{u_{+}}{p_{s}} \\
0 & 0 & \cdots & 0 & 1 \\
& & & & 0 \\
& \operatorname{Jac}(X) & & \vdots \\
& & & & 0
\end{array}\right]}
\end{aligned}
$$

Since $p_{s}=p_{+}$, we have completed the proof because the top row in the matrix above is $\left[\nabla l_{u}(p),-\frac{u_{+}}{p_{+}}\right]$.

The conormal variety of $X$ is defined to be the Zariski closure in $\mathbb{P}^{n} \times \mathbb{P}^{n}$ of the set

$$
N_{X}:=\overline{\left\{(p, q): q \perp T_{p} X_{r e g}\right\}} .
$$

To determine the defining equations of $N_{X}$, we let $M$ denote a $(k+1) \times(n+1)$ matrix that is an extended Jacobian whose top row is $\left[q_{0}, q_{1}, \ldots, q_{n}\right]$ and whose bottom rows are $\operatorname{Jac}(X)$. The defining equations of the conormal variety can be computed by taking the ideal generated by $f_{1}, \ldots, f_{k}$ and the $(c+1) \times(c+1)$-minors of $M$ and saturating by the $c \times c$-minors of $\operatorname{Jac}(X)$.

The dual variety $X^{*}$ is the projection of the conormal variety $N_{X}$ to the dual projective space $\mathbb{P}^{n}$ associated to the $q$-coordinates. To compute the equations of the dual variety, one eliminates the unknowns $p_{0}, p_{1}, \ldots, p_{n}$ from the equations defining $N_{X}$. For additional information on computing conormal varieties and dual varieties see [13].

Since $X^{\prime}$ is contained in a hyperplane defined by $H$, the dual variety of $X^{\prime}$ is known to be a cone of $X^{*}$ over the point $h=[-1:-1: \cdots:-1: 1][6]$ (Proposition 1.1). So $X^{* *}$ in $\mathbb{P}^{n+1}$ is given by

$$
\frac{X^{\prime *}=}{\left\{\left[q_{0}-b_{s}: q_{1}-b_{s}: \cdots: q_{n}-b_{s}: b_{s}\right]:\left[q_{0}: \cdots: q_{n}\right] \in X^{*}\right\}}
$$

It is easy to go between the coordinates of $X$ and coordinates of $X^{\prime}$ because there there is birational map between these two varieties. But there does not have to be a birational map between $X^{*}$ and $X^{\prime *}$. For this reason, the coordinates of the former are 
in $q_{0}, \ldots, q_{n}$, and the coordinates of the latter are in $b_{0}, \ldots, b_{n}, b_{s}$. Our notation is to let $q$ denote a point $\left[q_{0}: q_{1}: \cdots: q_{n}\right] \in X^{*}$ and to let $b$ denote a point $\left[b_{0}: b_{1}: \cdots: b_{n}\right.$ : $\left.b_{s}\right] \in X^{\prime *}$.

The next proposition shows that given the defining equations of $X^{*}$ in the unknowns $q_{0}, \ldots, q_{n}$, we can determine the defining equations of $X^{\prime *}$ in the unknowns $b_{0}, \ldots, b_{n}, b_{s}$ using the relations

$$
q_{0}=b_{0}+b_{s}, q_{1}=b_{1}+b_{s}, \ldots, q_{n}=b_{n}+b_{s} .
$$

Meaning, if $g\left(q_{0}, q_{1}, \ldots, q_{n}\right)$ vanishes on $X^{*}$, then $g\left(b_{0}+b_{s}, b_{1}+b_{s}, \ldots, b_{n}+b_{s}\right)$ vanishes on $X^{\prime *}$. Moreover, given the Jacobian of $X^{*}$, we can easily determine the Jacobian of $X^{\prime *}$ as well using the relations in (2).

Proposition 2. If $g_{1}(q), \ldots, g_{l}(q)$ define the variety $X^{*} \subset \mathbb{P}^{n}$ in coordinates $q_{0}, q_{1}, \ldots, q_{n}$, then the defining equations of $X^{* *}$ in coordinates $b_{0}, b_{1}, \ldots, b_{n}, b_{s}$ are

$$
\begin{gathered}
g_{1}\left(b_{0}+b_{s}, b_{1}+b_{s}, \ldots, b_{n}+b_{s}\right)=0 \\
\vdots \\
g_{l}\left(b_{0}+b_{s}, b_{1}+b_{s}, \ldots, b_{n}+b_{s}\right)=0 .
\end{gathered}
$$

Moreover, the Jacobian of $X^{\prime *}$ is given by

$$
\operatorname{Jac}\left(X^{\prime *}\right)=\left.\operatorname{Jac}\left(X^{*}\right)\right|_{\left(b_{0}+b_{s}, \ldots, b_{n}+b_{s}\right)}\left[\begin{array}{ccccc}
1 & & & & 1 \\
& 1 & & & \vdots \\
& & \ddots & & 1 \\
& & & 1 & 1
\end{array}\right] .
$$

Proof. The first part of proposition follows immediately from the relations in (2). By

$$
\left.\operatorname{Jac}\left(X^{*}\right)\right|_{\left(b_{0}+b_{s}, \ldots, b_{n}+b_{s}\right)}
$$

we mean evaluate the Jacobian of $X^{*}$ at $\left(b_{0}+b_{s}, \ldots b_{n}+b_{s}\right)$. Since the defining equations of $X^{*}$ are gotten by evaluating each $g_{i}(q)$ at $\left(b_{0}+b_{s}, \ldots b_{n}+b_{s}\right)$, it follows by the chain rule that $\operatorname{Jac}\left(X^{\prime *}\right)$ equals the desired matrix product.

Example 3. Consider $X$ in $\mathbb{P}^{3}$, a variety defined by

$$
f=2 p_{0} p_{1} p_{2}+p_{1}^{2} p_{2}+p_{1} p_{2}^{2}-p_{0}^{2} p_{12}+p_{1} p_{2} p_{12} .
$$

The Jacobian of $X$ and the defining polynomial $g(q)$ of the dual variety $X^{*}$ are

$$
\begin{array}{r}
\mathrm{Jac}(X)=\left[2 p_{1} p_{2}-2 p_{0} p_{12}, 2 p_{0} p_{2}+2 p_{1} p_{2}+p_{2}^{2}+p_{2} p_{12}\right. \\
\left.2 p_{0} p_{1}+p_{1}^{2}+2 p_{1} p_{2}+p_{1} p_{12},-p_{0}^{2}+p_{1} p_{2}\right]
\end{array}
$$


and

$$
\begin{array}{r}
g(q)=q_{0}^{4}-8 q_{0}^{2} q_{1} q_{2}+16 q_{1}^{2} q_{2}^{2}-8 q_{0}^{3} q_{12}+ \\
16 q_{0}^{2} q_{1} q_{12}+16 q_{0}^{2} q_{2} q_{12}-32 q_{0} q_{1} q_{2} q_{12}
\end{array}
$$

The variety $X^{\prime}$ is defined by the two equations,

$$
f(p)=0 \text { and } p_{s}=p_{0}+p_{1}+\cdots+p_{n},
$$

but the dual variety $X^{\prime *}$ is defined by one equation

$$
\begin{gathered}
g\left(b_{0}+b_{s}, b_{1}+b_{s}, b_{2}+b_{s}, b_{12}+b_{s}\right)= \\
\left(b_{0}+b_{s}\right)^{4}-8\left(b_{0}+b_{s}\right)^{2}\left(b_{1}+b_{s}\right)\left(b_{2}+b_{s}\right)+ \\
16\left(b_{1}+b_{s}\right)^{2}\left(b_{2}+b_{s}\right)^{2}-8\left(b_{0}+b_{s}\right)^{3}\left(b_{12}+b_{s}\right)+ \\
16\left(b_{0}+b_{s}\right)^{2}\left(b_{1}+b_{s}\right)\left(b_{12}+b_{s}\right)+ \\
16\left(b_{0}+b_{s}\right)^{2}\left(b_{2}+b_{s}\right)\left(b_{12}+b_{s}\right) \\
-32\left(b_{0}+b_{s}\right)\left(b_{1}+b_{s}\right)\left(b_{2}+b_{s}\right)\left(b_{12}+b_{s}\right) .
\end{gathered}
$$

The Jacobian of $X^{*}$ is

$$
\left[\begin{array}{c}
4 q_{0}^{3}-16 q_{0} q_{1} q_{2}-32 q_{12}\left(\frac{3}{4} q_{0}^{2}-q_{0} q_{1}-q_{0} q_{2}+q_{1} q_{2}\right) \\
-8 q_{0}^{2} q_{2}+32 q_{1} q_{2}^{2}+16 q_{0}^{2} q_{12}-32 q_{0} q_{2} q_{12} \\
-8 q_{0}^{2} q_{1}+32 q_{1}^{2} q_{2}+16 q_{0}^{2} q_{12}-32 q_{0} q_{1} q_{12} \\
-8 q_{0}^{3}+16 q_{0}^{2} q_{1}+16 q_{0}^{2} q_{2}-32 q_{0} q_{1} q_{2}
\end{array}\right]^{T}
$$

We get the Jacobian of $X^{*}$ by evaluating $\operatorname{Jac}\left(X^{*}\right)$ at $\left(b_{0}+b_{s}, \ldots b_{n}+b_{s}\right)$ and multiplying the evaluated $\operatorname{Jac}\left(X^{*}\right)$ on the right by the matrix

$$
\left[\begin{array}{lllll}
1 & & & & 1 \\
& 1 & & & 1 \\
& & 1 & & 1 \\
& & & 1 & 1
\end{array}\right]
$$

Now we are ready to state our first result.

Theorem 4. Fix an algebraic statistical model X. A point

$$
\left(\left[p_{0}: p_{1}: \cdots: p_{n}: p_{s}\right],\left[b_{0}: b_{1}: \cdots: b_{n}: b_{s}\right]\right) \in N_{X^{\prime}}
$$

satisfies the relation

$$
\left[p_{0} b_{0}: p_{1} b_{1}: \cdots: p_{n} b_{n}: p_{s} b_{s}\right]=\left[u_{0}: u_{1}: \cdots: u_{n}:-u_{+}\right]
$$

if and only if $\left[p_{0}: p_{1}: \cdots: p_{n}: p_{s}\right]$ is a critical point of $l_{u}^{\prime}(p)=p_{0}^{u_{0}} p_{1}^{u_{1}} \cdots p_{n}^{u_{n}} p_{s}^{-u_{+}}$on $X^{\prime}$. 
Proof. To determine critical points of $l_{u}^{\prime}(p)$ on $X^{\prime}$ we find when

$$
\nabla l_{u}^{\prime}(p)=\left[\partial l_{u}^{\prime} / \partial p_{0}: \cdots: \partial l_{u}^{\prime} / \partial p_{s}\right]
$$

is orthogonal to the tangent space of $X^{\prime}$ at the point $p$. This is the same as determining when

$$
\left(\left[p_{0}: p_{1}: \cdots: p_{s}\right], \nabla l_{u}^{\prime}(p)\right) \in N_{X^{\prime}} .
$$

As a point in projective space, we have

$$
\nabla l_{u}^{\prime}(p)=\left[\frac{u_{0}}{p_{0}}, \ldots, \frac{u_{n}}{p_{n}},-\frac{u_{+}}{p_{s}}\right]
$$

whenever $p_{0} p_{1} \cdots p_{s} \neq 0$. So we immediately have that a critical point of $l_{u}^{\prime}(p)$ satisfies the desired relations when we take the coordinate-wise product of $\left[p_{0}: p_{1}: \cdots: p_{s}\right]$ and $\nabla l_{u}^{\prime}(p)$.

With Lemma 2, Theorem 4 says that if $[p, b] \in N_{X^{\prime}}$ and the coordinate-wise product of $p$ and $b$ is

$$
\left[p_{0} b_{0}: \cdots: p_{n} b_{n}: p_{s} b_{s}\right]=\left[u_{0}: \cdots: u_{n}:-u_{+}\right]
$$

then $\left[p_{0}: \cdots: p_{n}\right]$ is a critical point of $l_{u}(p)$ on $X$.

Definition 5. The likelihood locus of $X$ for the data $u$ is defined as the set of points in $N_{X^{\prime}}$ satisfying the relations in (3), notated $L_{X}(u)$. We define $\mathcal{P}_{X}(u)$ and $\mathcal{B}_{X}(u)$ to be

$$
\mathcal{P}_{X}(u):=\left\{p:(p, b) \in L_{X}(u)\right\} \text { and } \mathcal{B}_{X}(u):=\left\{b:(p, b) \in L_{X}(u)\right\} .
$$

For additional clarification, note that points in $L_{X}(u)$ are contained in the conormal variety $N_{X^{\prime}} \subset \mathbb{P}^{n+1} \times \mathbb{P}^{n+1}$. These points are expressed as

$$
(p, b)=\left(\left[p_{0}: p_{1}: \cdots: p_{s}\right],\left[b_{0}: b_{1}: \cdots: b_{s}\right]\right) \in L_{X}(u) .
$$

In regards to ML degree, we have for generic choices of $u$

$$
\operatorname{MLdegree}(X)=\# L_{X}(u)=\# \mathcal{P}_{X}(u)=\# \mathcal{B}_{X}(u) \text {. }
$$

There are two corollaries to Theorem 4. The first corollary gives a bijection between critical points of $l_{u}^{\prime}(p)$ on $X^{\prime}$ and critical points of $l_{u}^{\prime}(b)$ on $X^{\prime *}$. The second corollary gives equations to determine critical points of $l_{u}^{\prime}(p)$ on $X^{\prime}$.

Corollary 1. There is a bijection between critical points of $l_{u}^{\prime}(p)$ on $X^{\prime}$ and critical points of $l_{u}^{\prime}(b)$ on $X^{* *}$ given by

$$
\left[p_{0} b_{0}: p_{1} b_{1}: \cdots: p_{n} b_{n}: p_{s} b_{s}\right]=\left[u_{0}: u_{1}: \cdots: u_{n}:-u_{+}\right] .
$$

Moreover, the product $l_{u}^{\prime}(p) l_{u}^{\prime}(b)$ remains constant over the set of critical points. 
Proof. The first part follows by noticing that the relation forces us to have

$$
\left[p_{0}: p_{1}: \cdots: p_{s}\right]=\left[u_{0} / b_{0}: u_{1} / b_{1}: \cdots:-u_{+} / b_{s}\right]
$$

which is also the gradient of $l_{u}^{\prime}(b)$. The second part follows as

$$
l_{u}^{\prime}(p) l_{u}^{\prime}(b)=u_{0}^{u_{0}} u_{1}^{u_{1}} \cdots u_{n}^{u_{n}}\left(-u_{+}\right)^{-u_{+}} .
$$

When $u_{0}, \ldots, u_{n}$ are positive integers, the bijection in Corollary 1 pairs positive critical points of $l_{u}^{\prime}(p)$ ordered by increasing likelihood with positive critical points of $l_{u}^{\prime}(b)$ ordered by decreasing likelihood!

Example 6. We will compute the ML degree of $X$ in Example 3 to be 3. We fix the data vector $\left(u_{0}, u_{1}, u_{2}, u_{12}\right)=\frac{1}{40}(2,13,5,20)$, and determine the points of $L_{X}(u)$

$\begin{array}{ccccc}p_{0} & p_{1} & p_{2} & p_{12} & p_{s} \\ .167493 & .242186 & .0532836 & .537037 & 1 \\ -.485608 & .632011 & .35886 & .494736 & 1 \\ -2.58189 & 5.56009 & 6.19312 & -8.17133 & 1 \\ b_{0} & b_{1} & b_{2} & b_{12} & b_{s} \\ .29852 & 1.34194 & 2.34594 & .931035 & -1 \\ -.102964 & .514232 & .348325 & 1.01064 & -1 \\ -.0193657 & .0584523 & .0201837 & -.0611895 & -1 .\end{array}$

The eliminants for $p_{0}, p_{1}, p_{2}$, and $p_{12}$ are

$$
\begin{gathered}
\left(100 p_{0}^{3}+290 p_{0}^{2}+74 p_{0}-21\right) \\
\left(62700 p_{1}^{3}-403430 p_{1}^{2}+314358 p_{1}-53361\right) \\
\left(1900 p_{2}^{3}-12550 p_{2}^{2}+4886 p_{2}-225\right) \\
\left(62700 p_{12}^{3}+447650 p_{12}^{2}-511962 p_{12}+136125\right) .
\end{gathered}
$$

The eliminants for $b_{0}, b_{1}, b_{2}, b_{12}$ of $L_{X}(u)$ are

$$
\begin{gathered}
\left(1680 b_{0}^{3}-296 b_{0}^{2}-58 b_{0}-1\right), \\
\left(34151040 b_{1}^{3}-65386464 b_{1}^{2}+27271868 b_{1}-1377519\right), \\
\left(28800 b_{2}^{3}-78176 b_{2}^{2}+25100 b_{2}-475\right), \\
\left(272250 b_{12}^{3}-511962 b_{12}^{2}+223825 b_{12}+15675\right) .
\end{gathered}
$$

Note that we are not saying that the ML degree of $X$ equals the ML degree of $X^{*}$. In general,

$$
\operatorname{MLdegree}(X) \neq \operatorname{MLdegree}\left(X^{*}\right) \text {. }
$$


The reason why equality fails is because

$$
b_{0}+b_{1}+\cdots+b_{n}-b_{s}
$$

does not vanish on $X^{\prime *}$. So there is no analogue of Lemma 2 involving $X^{\prime *}$ and $X^{*}$. In terms of previous literature, one should think of Corollary 1 as a generalization of Theorem 2 of [4].

Corollary 2. Fix a point $[p, b]$ of $N_{X^{\prime}}$ such that $p_{s} b_{s} \neq 0$. The following are equivalent:

1. The point $[p, b]$ is in $L_{X}(u)$.

2. For $i=0,1,2, \ldots, n$, the point $[p, b]$ satisfies

$$
u_{i} p_{s} b_{s}=-u_{+} p_{i} b_{i}
$$

3. There exists $\left[q_{0}: \cdots: q_{n}\right] \in X^{*}$ such that for $i=0,1, \ldots, n$

$$
u_{i} p_{s} b_{s}=-u_{+} p_{i}\left(q_{i}-b_{s}\right)
$$

Proof. It is immediate that parts 1 and 2 are equivalent. To see 2 and 3 are equivalent, recall $q_{i}=b_{i}+b_{s}$ for $i=0,1, \ldots, n$, from the definition of $X^{\prime *}$.

A consequence of these equations is that it removes the need for saturation by $p_{0} p_{1} \cdots p_{n}$ with Grobner basis computations that involve the likelihood equations whenever the $u_{i}$ are nonzero. In addition, if we restrict to the affine charts defined by $p_{s}=1$ and $b_{s}=-u_{+}$, then the condition $p_{s} b_{s} \neq 0$ is immediately satisfied.

\section{Dual Likelihood Equations}

In this section we will define a system of equations whose solutions are precisely

$$
\mathcal{B}_{X}(u)=\left\{b:(p, b) \in L_{X}(u)\right\} .
$$

Once we know the set $\mathcal{B}_{X}(u)$, we determine the critical points of $l_{u}(p)=p_{0}^{u_{0}} \cdots p_{n}^{u_{n}} / p_{+}^{u_{+}}$ on $X$ using Lemma 2 and Corollary 2, Concretely, if $b \in \mathcal{B}_{X}(u)$ then

$$
\left[p_{0}: \cdots: p_{n}\right]=\left[u_{0} / b_{0}: \cdots: u_{n} / b_{n}\right]
$$

is a critical point of $l_{u}(p)$ on $X$. For this reason we make the following definition.

Definition 7. The dual maximum likelihood estimation problem for the algebraic statistical model $X$ and data $u$ is to determine $\mathcal{B}_{X}(u)$, the set of critical points of $l_{u}^{\prime}(b)$ on $X^{\prime *}$. 
By Corollary 1, we find the critical points of $l_{u}^{\prime}(b)=b_{0}^{u_{0}} b_{1}^{u_{1}} \cdots b_{n}^{u_{n}} b_{s}^{-u_{+}}$on $X^{* *}$ to determine the set $\mathcal{B}_{X}(u)$. That is, we determine the points $b \in X^{* *}$ such that the gradient

$$
\nabla l_{u}^{\prime}(b)=\left[\frac{u_{0}}{b_{0}}: \frac{u_{1}}{b_{1}}: \cdots: \frac{u_{n}}{b_{n}}: \frac{-u_{+}}{b_{s}}\right]
$$

is orthogonal to the tangent space of $X^{\prime *}$ at $b$.

If $X^{*}$ has codimension $c$, which also means $X^{\prime *}$ has codimension $c$, then the dual likelihood equations are obtained by taking the sum of ideals generated by

- the polynomials defining $X^{\prime *}$, and

- the $(c+1) \times(c+1)$ minors of an extended Jacobian multiplied by a diagonal matrix with entries $b_{0}, b_{1}, \ldots, b_{n}, b_{s}$,

$$
\left[\begin{array}{c}
\nabla l_{u}(b) \\
\operatorname{Jac}\left(X^{\prime *}\right)
\end{array}\right]\left[\begin{array}{ccc}
b_{0} & & \\
& \ddots & \\
& & b_{s}
\end{array}\right],
$$

and saturating by the product of two ideals,

- the principal ideal generated by $b_{0} b_{1} \cdots b_{n} b_{s}$, and

- the ideal generated by the $c \times c$-minors of $\operatorname{Jac}\left(X^{\prime *}\right)$.

This gives us a formulation of the dual likelihood equations. Now we make some simplifications to these equations to get Theorem 8 ,

By Euler's relations of partial derivatives the columns of the matrix product in (4) are linearly dependent. Indeed the columns sum to zero, so we may drop the last column of the product without changing the rank.

By Proposition 2, if $g_{1}(q), \ldots, g_{l}(q)$ define the variety $X^{*}$, then the defining equations of $X^{\prime *}$ are

$$
\begin{gathered}
g_{1}\left(b_{0}+b_{s}, b_{1}+b_{s}, \ldots, b_{n}+b_{s}\right)=0 \\
\vdots \\
g_{l}\left(b_{0}+b_{s}, b_{1}+b_{s}, \ldots, b_{n}+b_{s}\right)=0 .
\end{gathered}
$$

and the Jacobian of $X^{\prime *}$ is

$$
\operatorname{Jac}\left(X^{\prime *}\right)=\left.\operatorname{Jac}\left(X^{*}\right)\right|_{\left(b_{0}+b_{s}, \ldots b_{n}+b_{s}\right)}\left[\begin{array}{ccccc}
1 & & & & 1 \\
& 1 & & & \vdots \\
& & \ddots & & 1 \\
& & & 1 & 1
\end{array}\right] .
$$

Since the last column of $\operatorname{Jac}\left(X^{\prime *}\right)$ is the sum of the first columns, it follows the dual likelihood equations can be reformulated by the next theorem. 
Theorem 8. Let $g_{1}(q), \ldots, g_{l}(q)$ define $X^{*} \subset \mathbb{P}^{n}$ with codimension c. Then, $\mathcal{B}_{X}(u)$ is variety of the ideal calculated by taking the sum of the ideals generated by

- $g_{1}\left(b_{0}+b_{s}, \ldots, b_{n}+b_{s}\right), \ldots, g_{l}\left(b_{0}+b_{s}, \ldots, b_{n}+b_{s}\right)$ and

- the $(c+1) \times(c+1)$ minors of

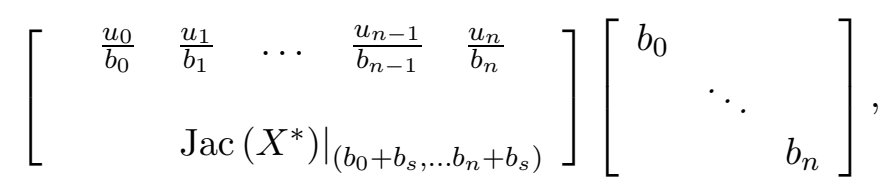

and saturating by the product of two ideals,

- the principal ideal generated by $b_{0} b_{1} \cdots b_{n} b_{s}$, and

- the ideal of $c \times c$-minors of $\left.\operatorname{Jac}\left(X^{*}\right)\right|_{\left(b_{0}+b_{s}, \ldots b_{n}+b_{s}\right)}$.

The point of Theorem 8 is that the dual likelihood equations define a homogeneous ideal in the polynomial ring $\mathbb{C}\left[b_{0}, b_{1}, \ldots, b_{n}, b_{s}\right]$ whose variety is $\mathcal{B}_{X}(u)$, the set of critical points of $l_{u}^{\prime}(b)$ on $X^{\prime *}$. Theorem 8 can be used to determine the ML degree of $X$ because $\# L_{X}(u)=\# \mathcal{B}_{X}(u)$.

Since Theorem 8 is constructive, we express it below as an algorithm.

Algorithm 9. Suppose $X^{*}$ in $\mathbb{P}^{n}$ has codimension $c$.

- Input: Polynomials $g_{1}(q), g_{2}(q), \ldots, g_{l}(q)$ defining $X^{*}$, and a vector $u \in \mathbb{N}^{n+1}$.

- Output: The ML degree of $X$.

\section{- Procedure:}

Step 1. Let $G_{q}$ be the ideal generated by $g_{1}(q), \ldots, g_{l}(q)$, and let $G_{b}$ be the ideal obtained by substituting $q_{0}, \ldots, q_{n}$ for $b_{0}+b_{s}, \ldots, b_{n}+b_{s}$, respectively, in the ideal $G_{q}$.

Step 2. Let $M_{b, u}$ denote the $(c+1)$-minors of (15) .

Step 3. Let $S_{b}$ be the ideal generated by the $c \times c$ minors of $\left.\operatorname{Jac}\left(X^{*}\right)\right|_{\left(b_{0}+b_{s}, \ldots b_{n}+b_{s}\right)}$. Step 4. Let $W_{b, u}$ be the saturation

$$
\left(M_{b, u}+G_{b}\right):\left(b_{0} b_{1} \cdots b_{n} b_{s} \cdot S_{b}\right)^{\infty}
$$

Step 5. Return the degree of $W_{b, u}$. 
Example 10. Let $X$ be defined by $f(p)=4 p_{0} p_{2}-p_{1}^{2}$ in $\mathbb{P}^{2}$. Then $X^{*}$ is defined by $g(q)=q_{0} q_{2}-q_{1}^{2}$ in the $\mathbb{P}^{2}$. So

$$
f(p)=\operatorname{det}\left[\begin{array}{cc}
2 p_{0} & p_{1} \\
p_{1} & 2 p_{2}
\end{array}\right] \text { and } g(q)=\operatorname{det}\left[\begin{array}{cc}
q_{0} & q_{1} \\
q_{1} & q_{2}
\end{array}\right] .
$$

The ML degree of $X$ is computed by taking the ideal generated by

- $g\left(b_{0}+b_{s}, b_{1}+b_{s}, b_{2}+b_{s}\right)=\left(b_{0}+b_{s}\right)\left(b_{2}+b_{s}\right)-\left(b_{1}+b_{s}\right)^{2}$, and

- $2 \times 2$ minors of

$$
\left[\begin{array}{ccc}
\frac{u_{0}}{b_{0}} & \frac{u_{1}}{b_{1}} & \frac{u_{2}}{b_{2}} \\
\left(b_{2}+b_{s}\right) & -2\left(b_{1}+b_{s}\right) & \left(b_{0}+b_{s}\right)
\end{array}\right]\left[\begin{array}{ccc}
b_{0} & & \\
& b_{1} & \\
& & b_{2}
\end{array}\right]
$$

and saturating by the product of two ideals

- the principal ideal $\left(b_{0} b_{1} b_{2} b_{s}\right)$ and

- the $1 \times 1$ minors of

$$
\left[\begin{array}{lll}
\left(b_{2}+b_{s}\right) & -2\left(b_{1}+b_{s}\right) & \left(b_{0}+b_{s}\right)
\end{array}\right] .
$$

We find that there is a unique critical point of $l_{u}^{\prime}(b)$ on $X^{\prime *}$ whose coordinates can be derived from the matrix equality

$$
\frac{1}{b_{s}}\left[\begin{array}{ll}
b_{0} & b_{1} \\
b_{1} & b_{2}
\end{array}\right]=\left[\begin{array}{cc}
\frac{4 u_{0} u_{+}}{\left(2 u_{0}+u_{1}\right)^{2}} & \frac{4 u_{1} u_{+}}{2\left(u_{1}+2 u_{1}\right)\left(2 u_{0}+u_{1}\right)} \\
\frac{4 u_{1} u_{+}}{2\left(u_{1}+2 u_{2}\right)\left(2 u_{0}+u_{1}\right)} & \frac{4 u_{2} u_{+}}{\left(2 u_{2}+u_{1}\right)^{2}}
\end{array}\right] .
$$

So by Corollary 2, the critical point of $l_{u}(p)$ on $X$ is given by

$$
\frac{1}{p_{s}}\left[\begin{array}{cc}
2 p_{0} & p_{1} \\
p_{1} & 2 p_{2}
\end{array}\right]=\frac{1}{2 u_{+}^{2}}\left[\begin{array}{c}
\left(2 u_{0}+u_{1}\right) \\
\left(u_{1}+2 u_{2}\right)
\end{array}\right]\left[\begin{array}{c}
\left(2 u_{0}+u_{1}\right) \\
\left(u_{1}+2 u_{2}\right)
\end{array}\right]^{T} .
$$

\subsection{Experimental Results}

In this section, we compare the standard formulation of solving the likelihood equations, Algorithm 6 of [10], to the dual formulation presented here, Algorithm 9. All computations in this subsection were done on a $2.8 \mathrm{GHz}$ Intel Core i7 MacBook Pro using 
Macaulay2.

$$
\begin{aligned}
& I_{1}=\left\langle q_{0}^{2}+2 q_{1}^{2}+3 q_{2}^{2}+5 q_{3}^{2}\right\rangle \\
& I_{2}=\left\langle q_{2}^{2}-q_{1} q_{3}, q_{1} q_{2}-q_{0} q_{3}, q_{1}^{2}-q_{0} q_{2}\right\rangle \\
& I_{3}=\left\langle q_{0}^{3}+q_{1}^{3}+q_{2}^{3}+q_{3}^{3}\right\rangle \\
& I_{4}=\left\langle 30 q_{0}^{2}+15 q_{1}^{2}+10 q_{2}^{2}+6 q_{3}^{2}\right\rangle \\
& I_{5}=\left\langle q_{1}^{2} q_{2}^{2}-4 q_{0} q_{2}^{3}-4 q_{1}^{3} q_{3}+18 q_{0} q_{1} q_{2} q_{3}-27 q_{0}^{2} q_{3}^{2}\right\rangle \\
& I_{6}=\left\langle q_{0}^{4}+q_{1} q_{2} q_{3}^{2}-q_{4}^{4}\right\rangle \\
& I_{7}=2 \times 2 \text { minors of }\left[\begin{array}{l}
2 q_{11}, q_{12}, q_{13} \\
q_{12}, 2 q_{22}, q_{23} \\
q_{13}, q_{23}, 2 q_{33}
\end{array}\right] \\
& I_{8}=2 \times 2 \text { minors of }\left[\begin{array}{l}
q_{11}, q_{12}, q_{13} \\
q_{12}, q_{22}, q_{23} \\
q_{13}, q_{23}, q_{33}
\end{array}\right] \\
& I_{10}=\left\langle\operatorname{det}\left[\begin{array}{c}
q_{0}, q_{1}, q_{2} \\
q_{1}, q_{2}, q_{3} \\
q_{2}, q_{3}, q_{4}
\end{array}\right]\right\rangle
\end{aligned}
$$

The second column in the table below is a list of ML degrees of varieties whose dual variety is given by the first column. The third column is the time (in seconds) it takes to calculate the ML degree using the standard formulation, while the fourth column is the time (in seconds) it takes to calculate the ML degree using the dual likelihood equations.

$\begin{array}{ccrr}X^{\star} & \text { ML degree } & \text { Standard } & \text { Dual } \\ I_{1} & 14 & 0.008 & 0.047 \\ I_{2} & 4 & 0.062 & 0.062 \\ \mathbf{I}_{3} & \mathbf{5 7} & \mathbf{1 6 6 . 8 7 2} & \mathbf{1 . 4 4 7} \\ I_{4} & 14 & 0.038 & 0.042 \\ I_{5} & 3 & 0.017 & 0.311 \\ I_{6} & 22 & 32.0657 & 13.594 \\ I_{7} & 13 & 4.808 & 33.489 \\ I_{8} & 6 & 2.349 & 13.842 \\ I_{10} & 3 & 1.0731 & 2.165\end{array}$

The most notable discrepancy is in row 3 in bold. In this case, the ideal of $X^{*}$ is generated by a cubic, but $X$ is generated by a degree 12 polynomial with 35 terms.

\subsection{Tensors}

To calculate new ML degrees when $X^{*}$ is not a complete intersection [Computation 12, we will work with an adjusted formulation of the dual likelihood equation. This formulation introduces codimension $X^{*}$ auxiliary unknowns (Lagrange multipliers). Also, instead of working with every generator of the ideal of $X^{*}$, we work with $\operatorname{codim}\left(X^{*}\right)$ 
generators. These generators should be chosen so that they define a reducible variety whose only irreducible component not contained in the coordinate hyperplanes is $X^{*}$.

Example 11. Consider $2 \times 2 \times 2$-tensors of the form $\left[p_{i j k}\right]$ with $i, j, k, \in\{0,1\}$. If $X$ is the hyperdeterminant of these tensors, then $X^{*}$ is defined by the $2 \times 2$ minors of all flattenings of the tensor $\left[q_{i j k}\right]$. The codimension of $X^{*}$ is 4 . The 4 flattenings below define $X^{*}$ after saturating by $q_{111}$.

$$
\begin{array}{ll}
g_{1}(q)=q_{011} q_{101}-q_{001} q_{111} & g_{2}(q)=q_{011} q_{110}-q_{010} q_{111} \\
g_{3}(q)=q_{001} q_{110}-q_{000} q_{111} & g_{4}(q)=q_{011} q_{101}-q_{001} q_{111}
\end{array}
$$

So by introducing auxiliary unknowns $\lambda_{0}, \lambda_{1}, \ldots, \lambda_{4}$ we create a square system of 12 equations in the homogeneous variable groups $\left(b_{000}, \ldots, b_{111}, b_{s}\right)$ and $\left(\lambda_{0}, \ldots, \lambda_{4}\right)$.

$$
\begin{gathered}
g_{1}=\left(b_{011}+b_{s}\right)\left(b_{101}+b_{s}\right)-\left(b_{001}+b_{s}\right)\left(b_{111}+b_{s}\right) \\
g_{2}=\left(b_{011}+b_{s}\right)\left(b_{110}+b_{s}\right)-\left(b_{010}+b_{s}\right)\left(b_{111}+b_{s}\right) \\
g_{3}=\left(b_{001}+b_{s}\right)\left(b_{110}+b_{s}\right)-\left(b_{000}+b_{s}\right)\left(b_{111}+b_{s}\right) \\
g_{4}=\left(b_{011}+b_{s}\right)\left(b_{101}+b_{s}\right)-\left(b_{001}+b_{s}\right)\left(b_{111}+b_{s}\right) \\
{\left[\lambda_{0}, \lambda_{1}, \lambda_{2}, \ldots, \lambda_{4}\right]\left[\begin{array}{c}
\nabla l_{u}^{\prime}(b) \\
\operatorname{Jac}(g)
\end{array}\right]\left[\begin{array}{cc}
b_{000} & \\
& \ddots \\
& b_{111}
\end{array}\right]=0 .}
\end{gathered}
$$

The solutions with $\lambda_{0} b_{s} \neq 0$ give the critical points. We find that there are 13 critical points of $l_{u}^{\prime}(b)$ on $X^{*}$, agreeing with [5], page 53 .

The next example is a new computational result to determine the ML degree of a hyperdeterminant.

Computation 12. Let $X$ denote the hyperdeterminant of $2 \times 2 \times 3$ tensors of the form $\left[p_{i j k}\right]$ for $i \in\{0,1\}, j \in\{0,1\}, k \in\{0,1,2\}$. Then the ML degree of $X$ is 71 .

Proof. The variety $X$ is dual to the variety $X^{*}$ defined by the $2 \times 2$-minors of the flattenings of the $2 \times 2 \times 3$ tensor $\left[q_{i j k}\right]$ with $i \in\{0,1\}, j \in\{0,1\}, k \in\{0,1,2\}$. The variety $X^{*}$ has codimension 7 , degree 12 , and 24 generators. We consider 7 of the 24 generators,

$$
\begin{aligned}
& g_{1}(q)=q_{102} q_{111}-q_{101} q_{112} \\
& g_{2}(q)=q_{102} q_{110}-q_{100} q_{112} \\
& g_{3}(q)=q_{002} q_{111}-q_{001} q_{112} \\
& g_{4}(q)=q_{012} q_{102}-q_{002} q_{112} \\
& g_{5}(q)=q_{012} q_{111}-q_{011} q_{112} \\
& g_{6}(q)=q_{012} q_{110}-q_{010} q_{112} \\
& g_{7}(q)=q_{002} q_{110}-q_{000} q_{112}
\end{aligned}
$$


such that when saturated by $q_{112}$ we recover the dual variety $X^{*}$. We solve the following square system of equations: the seven equations

$$
g_{1}\left(b_{0}+b_{s}, \ldots, b_{n}+b_{s}\right)=\cdots=g_{7}\left(b_{0}+b_{s}, \ldots, b_{n}+b_{s}\right)=0
$$

and the 12 equations

$$
\left[1, \lambda_{1}, \lambda_{2}, \ldots, \lambda_{7}\right] M\left[\begin{array}{lll}
b_{101} & & \\
& \ddots & \\
& & b_{112}
\end{array}\right]=0
$$

with $M$ being (6) where $\left(q_{101}, \ldots, q_{112}\right)=\left(b_{101}+b_{s}, \ldots, b_{112}+b_{s}\right)$ and $u$ consisting of random complex numbers (random positive integers) to determine the ML degree of $X$ numerically (symbolically).

$$
\left[\begin{array}{cccccccccccc}
\frac{u_{101}}{b_{101}} & u_{011} & \frac{u_{100}}{b_{011}} & \frac{u_{010}}{b_{010}} & \frac{u_{001}}{b_{001}} & \frac{u_{000}}{b_{000}} & \frac{u_{002}}{b_{002}} & \frac{u_{012}}{b_{012}} & \frac{u_{102}}{b_{102}} & \frac{u_{110}}{b_{110}} & \frac{u_{111}}{b_{111}} & \frac{u_{112}}{b_{112}} \\
-q_{112} & 0 & 0 & 0 & 0 & 0 & 0 & 0 & q_{111} & 0 & q_{102} & -q_{101} \\
& -q_{112} & 0 & 0 & 0 & 0 & 0 & q_{111} & 0 & 0 & q_{012} & -q_{011} \\
& & -q_{112} & 0 & 0 & 0 & 0 & 0 & q_{110} & q_{102} & 0 & -q_{100} \\
& & & -q_{112} & 0 & 0 & 0 & q_{110} & 0 & q_{012} & 0 & -q_{010} \\
& & & & -q_{112} & 0 & q_{111} & 0 & 0 & 0 & q_{002} & -q_{001} \\
& & & & & -q_{112} & q_{110} & 0 & 0 & q_{002} & 0 & -q_{000} \\
& & & & & & -q_{112} & q_{102} & q_{012} & 0 & 0 & -q_{002}
\end{array}\right]
$$

The ML degree 71 was obtained using exact methods in Macaulay2 in 10,943 seconds and using numerical methods in Bertini in 5,796 seconds. Both computations were performed on the UC Berkeley server apppsa which has four 16-core 2.3GHz AMD Opteron 6276 CPUs. The Bertini computation was done in parallel using 20 of the 64 cores.

One could have attempted to compute the number 71 using Algorithm 6 of [10]. However, to do so, we must have the defining equations of $X$. We were not able to compute these equations ourselves, but the hyperdeterminant of $2 \times 2 \times 3$ tensors is listed on page 7 of [2]. This is a degree 6 polynomial with 66 terms. We were unable to determine the 71 with the standard likelihood equations and with the Lagrange likelihood equations (page 4 of [8]).

The next interesting case is when $X$ is the hyperdeterminant of $2 \times 2 \times 2 \times 2$ tensors. In this case, $X$ is defined by a polynomial of degree 24 in 16 unknowns that has $2,894,276$ terms [11]. There is no way we will be able to effectively write down the standard likelihood equations for $X$. However, it's dual variety $X^{*}$ is a binomial ideal consisting of the $2 \times 2$-minors of all of its flattenings, and we may have a chance of solving the dual likelihood equations both numerically and symbolically. 


\section{The Dual MLE Problem vs Maximum Likelihood Duality}

In this section we introduce an example and show how the results presented in this paper fit in context with previous work on Maximum Likelihood Duality. In [4, 9] the notion of Maximum likelihood duality (ML-duality) was introduced. ML-duality gave a bijection between critical points of $l_{u}(p)$ on two different statistical models.

Definition 13. A pair of algebraic statistical models $X$ and $Y$ in $\mathbb{P}^{n}$ are said to be $M L$-dual if for generic $u$ there is an involutive bijection between points of $L_{X}(u)$ and points of $L_{Y}(u)$. Moreover, this bijection pairs points of $L_{X}(u)$ with points of $L_{Y}(u)$ such that the coordinate-wise product of each pair can be expressed in terms of the data $u$ alone.

Example 14. Suppose $r \leq m \leq n$, and let $V_{m, n, r}$ denote the Zariski closure in $\mathbb{P}^{m n-1}$ of rank $r$ matrices of the form

$$
\left[\begin{array}{cccc}
p_{11} & p_{12} & \ldots & p_{1 n} \\
p_{21} & p_{22} & & \\
\vdots & & \ddots & \\
p_{m 1} & & & p_{m n}
\end{array}\right] .
$$

Then $V_{m, n, r}^{*}$ is known to be the Zariski closure in $\mathbb{P}^{m n-1}$ of rank $m-r$ matrices of the form

$$
\left[\begin{array}{cccc}
q_{11} & q_{12} & \ldots & q_{1 n} \\
q_{21} & q_{22} & & \\
\vdots & & \ddots & \\
q_{m 1} & & & q_{m n}
\end{array}\right] .
$$

Fix a choice of $m, n, r$. If we take $X=V_{m, n, r}$, then points in $X^{\prime}$ will be represented as

$$
\left[p_{i j}: p_{s}\right] \in X^{\prime} \subset \mathbb{P}^{m n}
$$

and points in $X^{\prime *}$ will be represented as

$$
\left[b_{i j}: b_{s}\right] \in X^{\prime *} \subset \mathbb{P}^{m n} .
$$

With Corollary 1, it follows there is a bijection between $\mathcal{P}_{X}(u)$ and $\mathcal{B}_{X}(u)$. On the other hand, by Theorem 1 in [4] we know that $V_{m, n, r}$ and $V_{m, n, m-r}$ are ML-dual. This means if we take $Y$ to be $V_{m, n, m-r}$ there is an involutive bijection between critical points $L_{X}(u)$ and $L_{Y}(u)$ for generic choices of $u$. In particular, the bijection is such that the coordinate-wise product of the paired points is

$$
\left(\left[\frac{u_{i+} u_{+j} u_{i j}}{u_{++}^{3}}: 1\right],\left[\frac{u_{i j} u_{++}}{u_{i+} u_{+j}}: 1\right]\right) \in \mathbb{P}^{m n} \times \mathbb{P}^{m n} .
$$

Here $u_{++}:=\sum_{i, j} u_{i j}, u_{i+}:=\sum_{j} u_{i j}$, and $u_{+j}:=\sum_{i} u_{i j}$, and likewise for $p_{++}, p_{i+}, p_{+j}$. 


\section{Conclusion}

In this paper we have given an elegant formulation of the MLE problem involving conormal varieties. This formulation allows us to avoid the computation of saturation by the product of unknowns. We also define the dual likelihood equations that allow us to compute critical points on $X$ even if we only know the defining equations of its dual $X^{*}$ [Algorithm 9]. The important feature of the dual likelihood equations comes from the fact that the defining equations of $X$ may be too difficult to work with. In addition, we showed that if we solve the dual equations, we can recover the critical points on $X$ [Theorem 4. More broadly, we showed that if there is a bijection between critical points of a function restricted to a variety and critical points of a monomial restricted

to a different variety, then we can formulate a new set of "dual" equations to determine these points.

\section{Acknowledgements}

The author would like to thank Elizabeth Gross, Kim Laine, Zvi Rosen, and Bernd Sturmfels for their comments and suggestions to improve earlier versions of the paper.

\section{References}

[1] D. J. Bates, J. D. Hauenstein, A. J. Sommese, and C. W. Wimpier. Bertini: Software for numerical algebraic geometry. Available at https://bertini.nd.edu/.

[2] M. R. Bremner. A hyperdeterminant for 2 x 2 x 3 arrays. arXiv:1106.2988, 2011.

[3] F. Catanese, S. Hoşten, A. Khetan, and B. Sturmfels. The maximum likelihood degree. Amer. J. Math., 128(3):671-697, 2006.

[4] J. Draisma and J. I. Rodriguez. Maximum likelihood duality for determinantal varieties. To appear in International Mathematics Research Notices, 2013.

[5] M. Drton, B. Sturmfels, and S. Sullivant. Lectures on algebraic statistics, volume 39 of Oberwolfach Seminars. Birkhäuser Verlag, Basel, 2009.

[6] L. Ein. Varieties with small dual varieties, i. Inventiones mathematicae, 86(1):63$74,1986$.

[7] D. R. Grayson and M. E. Stillman. Macaulay2, a software system for research in algebraic geometry. Available at http://www.math.uiuc.edu/Macaulay2/

[8] E. Gross and J. I. Rodriguez. Maximum likelihood geometry in the presence of data zeros. arXiv:1310.4197, 2014. 
[9] J. Hauenstein, J. I. Rodriguez, and B. Sturmfels. Maximum likelihood for matrices with rank constraints. To appear in the Journal of Algebraic Statistics, 2013.

[10] S. Hoşten, A. Khetan, and B. Sturmfels. Solving the likelihood equations. Found. Comput. Math., 5(4):389-407, 2005.

[11] P. Huggins, B. Sturmfels, J. Yu, and D. S. Yuster. The hyperdeterminant and triangulations of the 4-cube. Math. Comput., 77(263):1653-1679, 2008.

[12] J. Huh. The maximum likelihood degree of a very affine variety. Compos. Math., 149(8):1245-1266, 2013.

[13] P. Rostalski and B. Sturmfels. Dualities. In Semidefinite optimization and convex algebraic geometry, volume 13 of MOS-SIAM Ser. Optim., pages 203-249. SIAM, Philadelphia, PA, 2013. 